\title{
Wearable Devices to Control Objects in Virtual Reality
}

\author{
Shyh-Shing Perng, ${ }^{1 *}$ Nien-Tsu Hu, ${ }^{2}$ Pu-Sheng Tsai, ${ }^{3}$ Ter-Feng Wu, ${ }^{1}$ and Jen-Yang $\mathrm{Chen}^{2}$ \\ ${ }^{1}$ Department of Electrical Engineering, National Ilan University, Yilan 26047, Taiwan, ROC \\ ${ }^{2}$ Department of Electronic Engineering, Ming Chuan University, Taoyuan 33348, Taiwan, ROC \\ ${ }^{3}$ Department of Electronic Engineering, China University of Science and Technology, Taipei 11581, Taiwan, ROC
}

(Received December 27, 2019; accepted March 12, 2020)

Keywords: wearable device, virtual reality, Unity 3D, electronic compass, three-axis accelerometer

In this study, we focus on the design of a wearable inertial navigation device that is based on a MEMS chip and a Bluetooth wireless transmitting module and that uses the object-controlling technology of the Unity game engine to write a game script for a treasure hunt in an ancient Chinese virtual reality (VR) world. The wearable device proposed in this paper utilizes an ATmega168 chip as the main controller, which cooperates with a three-axis accelerometer and gyroscopic sensor to detect the forward, backward, and rotating movements of game players. The movement data are transmitted to the Unity 3D game engine using Bluetooth, and the game software can then control the first-person character. The interactive elements between reality and the VR world combined as described in this paper can deliver a new experience to games, driving simulators, medical surgery, and even a variety of commercial situations. Experimental results show that the performance of the proposed wearable device is stable.

\section{Introduction}

Wearable devices ${ }^{(1-4)}$ are electronic devices ${ }^{(5-7)}$ that can be worn on the body. They utilize various sensors to collect related data, including physiological information (such as heart rate, body temperature, blood pressure, blood oxygen, and steps taken), environmental data (such as temperature, humidity, $\mathrm{CO}_{2}$, and light), and motion perception (in which inertial sensing elements gauge complex human actions and behaviors). Wearable devices can have a greater range of application when connected to a mobile phone through a communication interface such as Bluetooth or when wirelessly connected to a cloud platform through Wi-Fi for big data processing. Generally, areas of application for wearable devices include leisure and entertainment, health care and health management, information transmission, and environmental monitoring. The most successful wearable products on the market are smart wristbands and smart watches, many of which have built-in accelerometers and gyroscopic sensors that calculate and analyze the collected sensing data and then provide wearers with statistical information on various aspects of life, such as running, walking, lying, and standing. They can even be used to detect the quality of sleep and provide a completely different "quantitative" experience for users: long-term health management based on statistics from more realistic data *Corresponding author: e-mail: ssperng@ems.niu.edu.tw https://doi.org/10.18494/SAM.2020.2789 
and long-term records. Examples of wearable devices include iWatch (the first Apple wearable device), the ASUS ZenWatch, the LG G Watch R, and the Sony SmartWatch 3.

The purpose of this study was to construct a treasure hunt game script in an ancient Chinese virtual reality (VR) ${ }^{(8-10)}$ environment by combining VR and wearable devices. Research motivations included (1) to demonstrate the potential of the ATmega168 core chip and sensors, such as a three-axis accelerometer and gyroscopic sensor, which are incorporated into a watch-type wearable device that can detect forward, left-turning, and right-turning actions of game players and whose functions are similar to those of the Mi Band; (2) to study the technology that the Unity game engine uses to control objects in VR and establish a new milestone for window animation in a human-machine interface; and (3) to cultivate the capabilities of a motion-sensing interactive multimedia device, focusing in particular on enhancing the functions of the wireless transferring module to make future cloud processing possible.

In this paper, we emphasize the inertial sensor components of the wearable device that can detect physical movements, allowing players to immerse themselves in a VR world and interact with game scripts in a timely manner, making them feel as if they are personally on the scene. To achieve game themes with realistic effects, we applied 3ds Max modeling software to produce various object models, including ancient Chinese architecture (huts) and ancient Chinese pavilions, which were imported into the Unity 3D software to design a VR world with ancient China as the background, thus allowing players to enter the VR world, where virtual characters engaged in a treasure hunt can be controlled in an interactive somatosensory fashion. This will allow players to feel as if they are personally on the scene.

\section{System Architecture}

The system is composed of three subunits: a personal computer, a Bluetooth wireless receiver, and a wearable device, as shown in Fig. 1. The wearable device is bound to the arm, and the system determines the position and direction of body movement or rotation. This device contains a three-axis accelerometer, an electronic compass, a gyroscope, and a Bluetooth

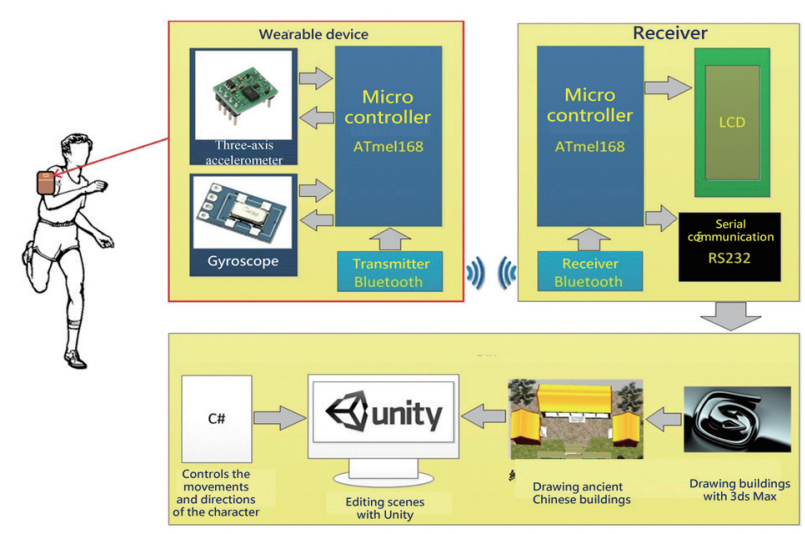

Fig. 1. (Color online) Block diagram of the system. 
transmitting module. The tilt detection of the three-axis accelerometer can sense movements of the arm, which indicate how the user is moving. The electronic compass can sense the user's position (the angle between the user and north), and the gyroscope can identify the rotation of the player. The Bluetooth transmitting module transmits the position and rotation signal to the signal receiver, and the RS232 serial communicator then sends the signal to the personal computer. The Unity game engine receives the signal in real time and writes a script in the C\# programming language to control the first-person character controller in VR.

\subsection{Transmitting board for the wearable device}

The Bluetooth transmitting board for the wearable device is shown in Fig. 2 and the elements of the transmitting board are shown in Fig. 3. Elements in the figure include (A) ATmega168 microcontroller, (B) three-axis accelerometer, (C) electronic compass, (D) Bluetooth communication module, $(\mathrm{E})$ gyroscope, $(\mathrm{F})$ battery box $(6 \mathrm{~V})$, and $(\mathrm{G})$ voltage regulator. The three-axis accelerometer tilts to detect body movements, and the gyroscope senses the user's speed to detect rotations of the body.

\subsection{Receiving board for the wearable device}

The data are transmitted from the Bluetooth transmitting module to the Bluetooth receiver, which is shown in Fig. 4. In the figure, the modules in the circuit include (A) backlit $16 \times 4$ text-type liquid crystal display (LCD) module, (B) RS232 female connector, (C) MAX232 level conversion chip, (D) Atmel 168 microcontroller, and (E) Bluetooth receiving module. The RS232 serial communicator then transmits the three-axis accelerometer and electronic compass data received from the wearable device to the Unity game engine.

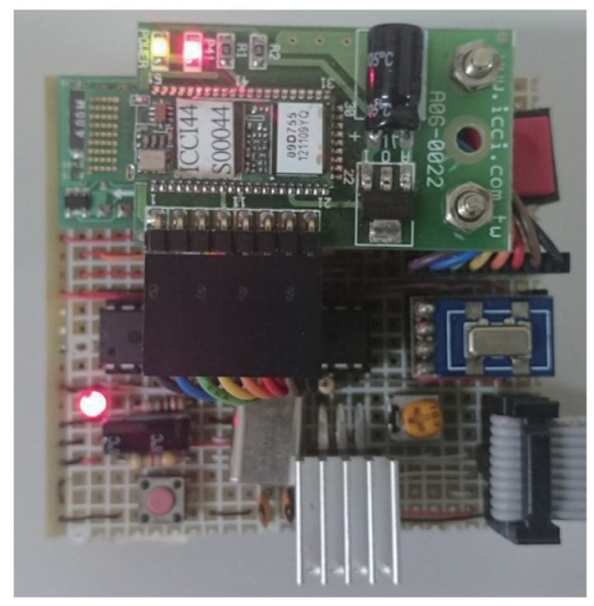

Fig. 2. (Color online) Appearance of the Bluetooth transmitting board.

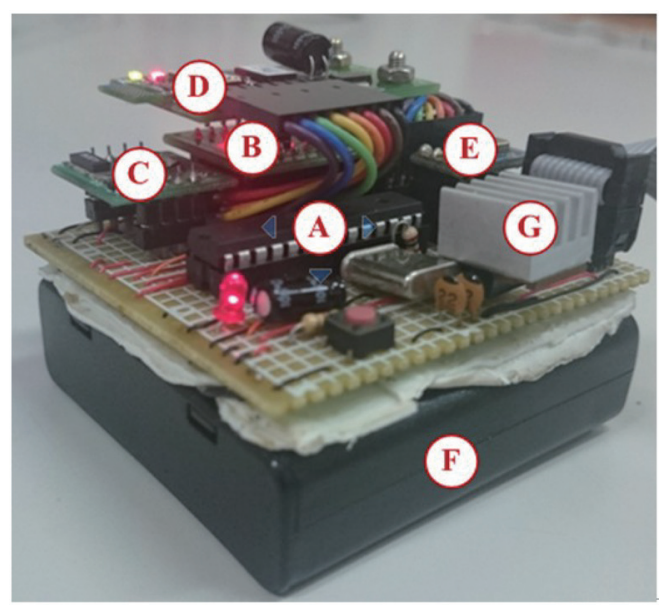

Fig. 3. (Color online) Elements of the Bluetooth transmitting board. 


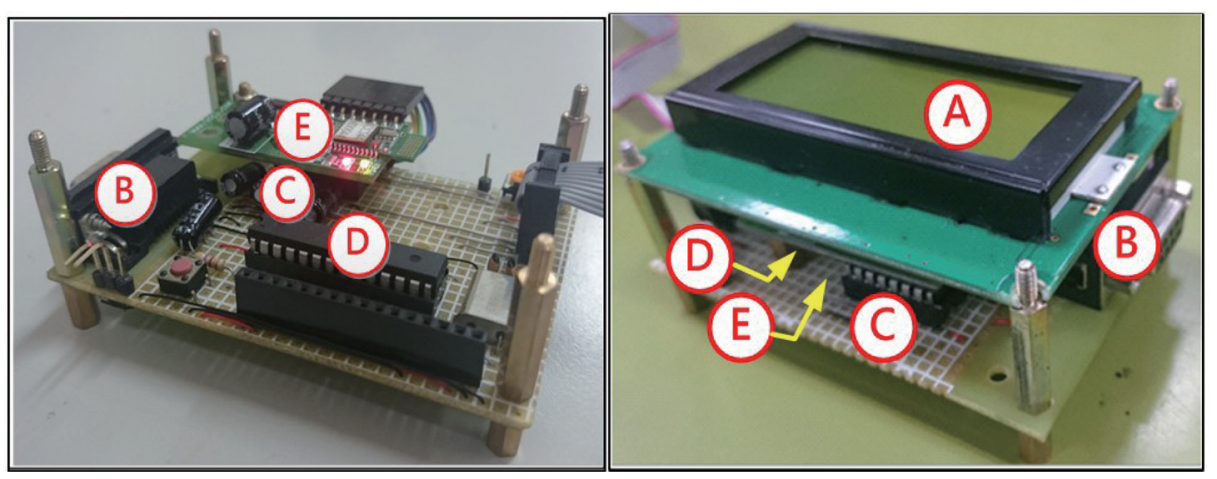

Fig. 4. (Color online) Appearance and elements of the Bluetooth receiving board for the wearable device.

\subsection{Unity 3D working platform}

Unity 3D, a free game development engine, has a variety of objects and materials built in. In addition to rich and simple art resources, it supports cross-platform development for Windows, macOS, Android, and other operating systems to improve product promotion. Unity's userfriendly interface significantly simplifies game development so that users can easily develop games. Modeling materials can be imported from Maya and 3ds Max into Unity 3D, and Unity 3D scripts can be written using JavaScript or C\# to design the interaction program for controlling objects in a scene.

\section{Modeling with 3ds Max}

3ds Max is an application with powerful functions for 3D modeling, animation production, shading, and rendering. It is widely used in visual effects, interior design, character animation, game development, and other fields, as shown in Fig. 5. Its functions include basic modeling, curve modeling, polygon modeling, model modification, texture map settings, light settings, photography animation, and rendering.

\section{Scene Creation with Unity 3D}

\subsection{Game scene browsing}

Figure 6 shows a game scene designed in Unity, Fig. 7 shows a sunset view of the Wudang Mountains in Hubei, and Fig. 8 shows a game scene based on the Wudang Mountains designed in this study.

The purpose of this study was to design a wearable device that uses inertial navigation and to develop a treasure hunting game script with an ancient Chinese background using technology to control objects in VR. The overall design of the scene is shown in Fig. 9. For this study, 3ds Max was used to build a variety of 3D models, which were imported into the Unity game engine software. The 3D models include ancient pavilions (marked as (5) in Fig. 9), Guiyuan Temple (6), 


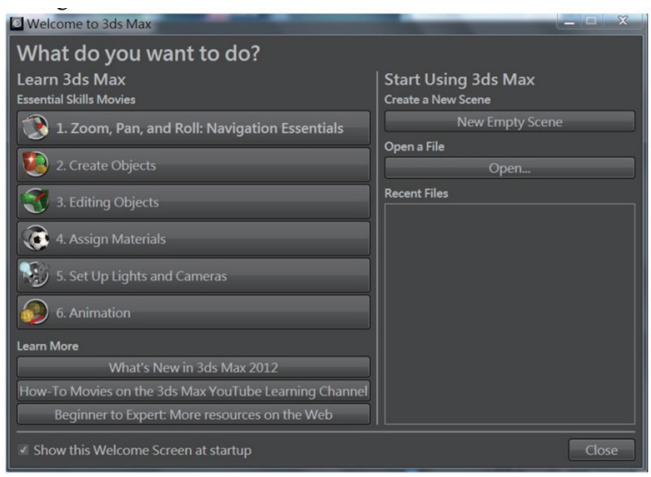

Fig. 5. (Color online) Powerful functions of $3 \mathrm{ds}$ Max.

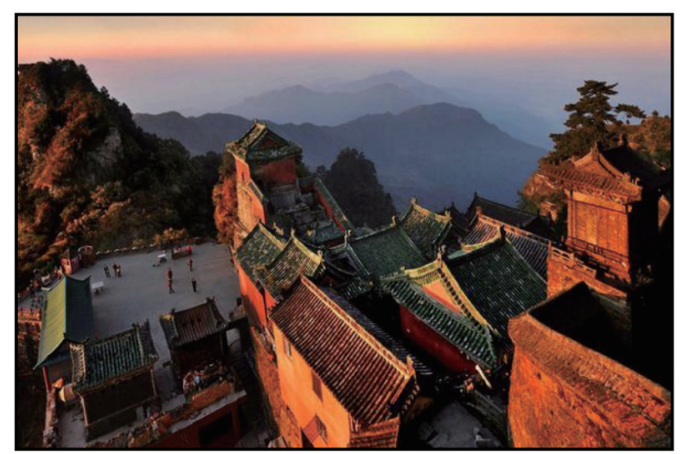

Fig. 7. (Color online) Hubei Wudang Mountains at dusk.

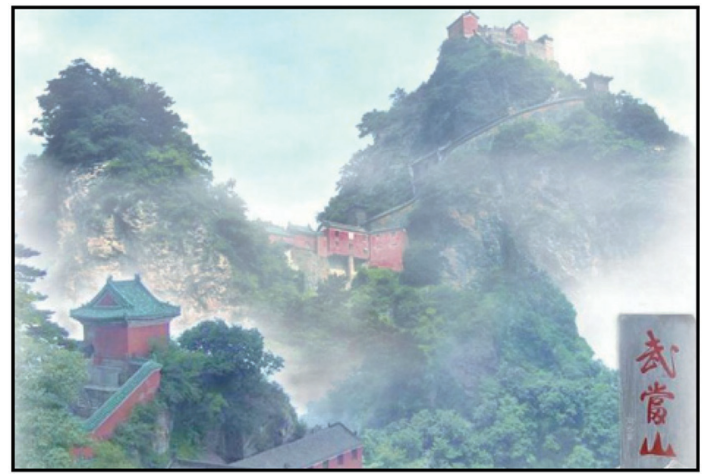

Fig. 6. (Color online) Hubei Wudang Mountains in the early morning.

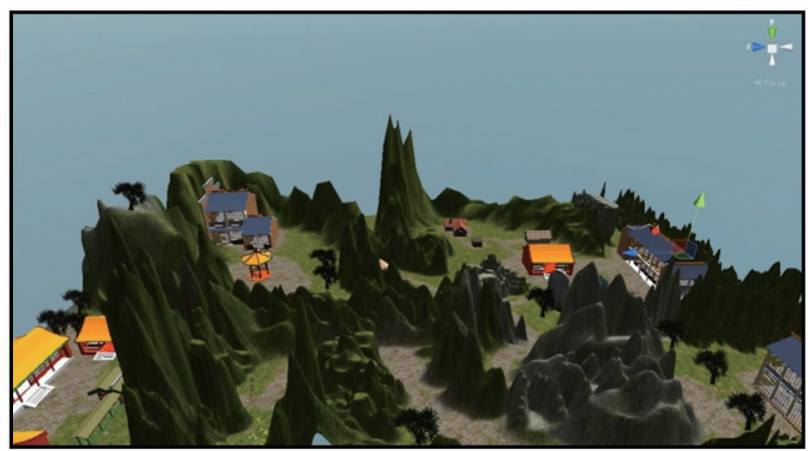

Fig. 8. (Color online) Game scene simulating the Hubei Wudang Mountains.

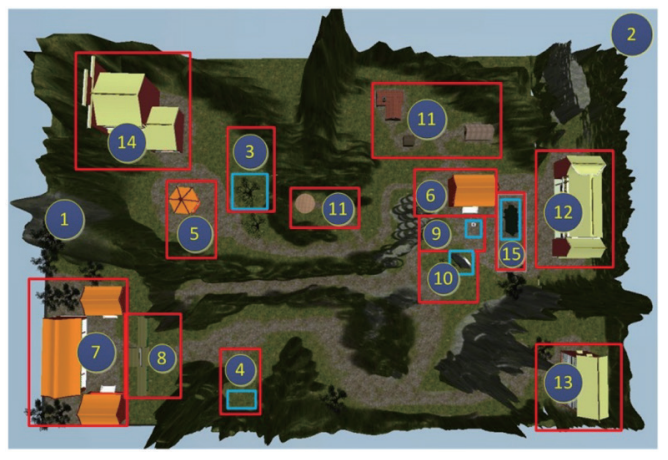

Fig. 9. (Color online) Game scene of hunting treasure in ancient China.

an ancient palace (7), a palace gate (8), a Wudang stone tablet (10), a thatched stone house (11), Guhua courtyard (12), an attic wing (13), and a waterside pavilion (14). The game scene also includes features created using built-in functions from Unity such as terrain creation (landscape, marked as (1)), sky rendering (effect, (2)), tree planting (objects, (3)), lawn planting (objects, (4)), a first-person controller (9), and a pool (15). 
The story script takes the first-person perspective of Zhang Wuji, the famous protagonist of "The Heaven Sword and Dragon Saber". At the beginning of the game, Zhang Wuji completes the closed-door practice of martial arts and comes out from Guiyuan Temple (6). When the protagonist steps out of the temple, he finds a Wudang stone tablet (10) standing beside him. The stone tablet is engraved with a maxim: "Dragon Sword, the master hero. Order the world, no one dares to reject. When the Heavenly Sword comes out, nothing can compete with it." After reading the maxim, Zhang Wuji must begin to look for the Dragon Saber and the Heaven Sword, which have been hidden in the ancient Chinese palace (7). To win the game, the protagonist must travel from Guiyuan Temple (6) to the ancient Chinese palace (7), collect the saber and sword, and strike them together to obtain the power of the Yue Fei martial arts, which is hidden within the Dragon Saber.

\subsection{Unity 3D collision detection}

The system uses the first-person controller of the Unity game engine. The wearable device worn on the arm of the game player sends the arm-swing motion and body rotation signals, detected by the three-axis accelerometer, gyroscope, and compass, to the Unity gaming software using Bluetooth wireless transmission. The first-person controller of the game can then be controlled to move forward, turn left, or turn right. However, when the game character moves and encounters obstacles such as trees, buildings, hills, and cliffs, the player must overcome the obstacles by continuing to move forward or turn around, which is different from the physical laws of the real world. Unity 3D provides a set of powerful collision detection algorithms. As long as objects are attached to colliders and the collision relationships among all objects are properly set, the first-person controller cannot pass through trees or buildings. Of course, bullets that go through walls can also be made. In addition, a collider can be used in shooting-based games as a tool to determine whether a target has been shot. Unity offers various types of colliders, including box colliders, sphere colliders, capsule colliders, and mesh colliders. Figure 10 shows collision detection results of the first-person controller. Figures 11 and 12 show a mesh collider attached to a robot arm to simulate the action of object clipping.

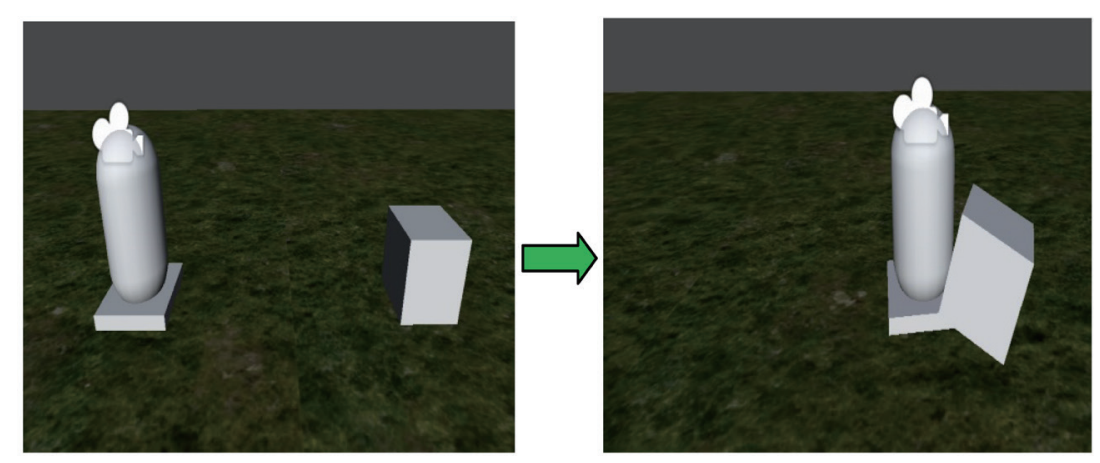

Fig. 10. (Color online) First-person collision detection. 


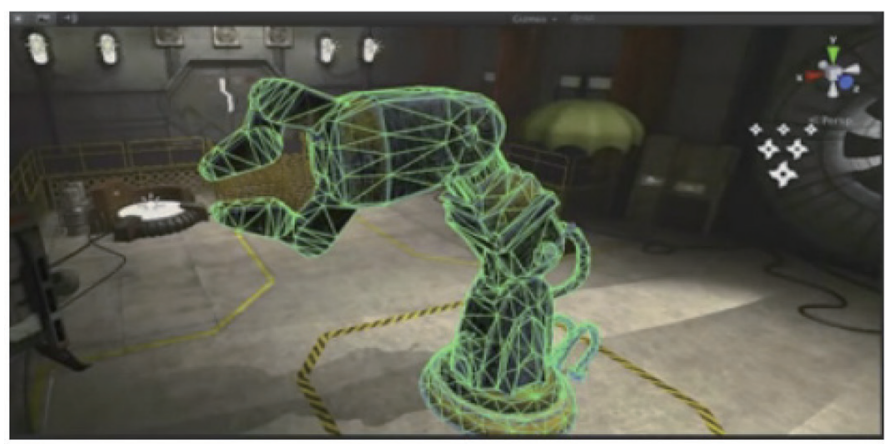

Fig. 11. (Color online) Mesh collider attached to a robot arm.

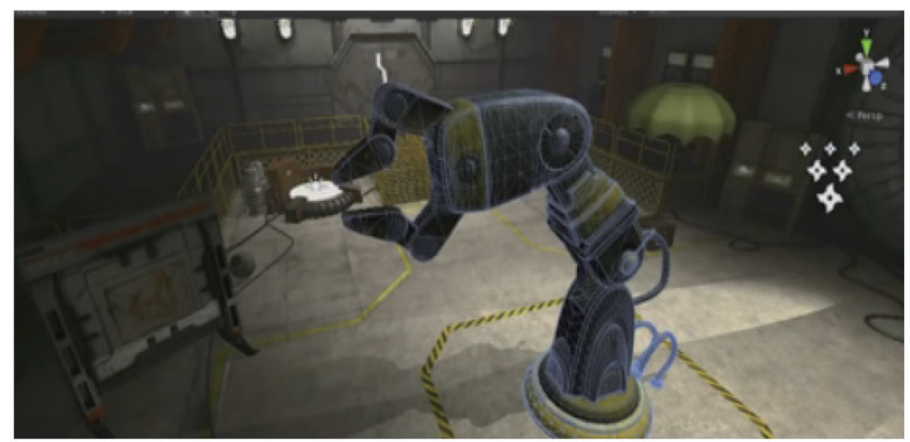

Fig. 12. (Color online) Simulated object clipping.

\section{Functional Tests and Experiment Results}

Figure 13 shows a player placing the wearable device on the sleeve of his arm. Figure 14 shows LCD screenshots with data that the Bluetooth receiver receives from the wearable device. After being powered to reset, the receiver was initialized and, after five seconds, it received the signal from the three-axis accelerometer and electronic compass. The LCD panel of the Bluetooth receiver displays information including the degree of inclination on the $Y$-axis, the arm-swinging time, the angle between the player and north, and whether the game player was rotating.

Figures 15 and 16 show the arm-swing actions of the game player. The wearable device senses arm movements of the game player and uses the Bluetooth wireless transmission module to transmit the data to the Bluetooth receiver. The RS232 chipset from the Bluetooth receiver then transmits the data to the Unity game engine, which writes a program in C\# allowing the first-person controller in the Unity screen to move forward, as shown in Figs. 17 and 18.

The purpose of this study was to use an eight-bit RISC microcontroller chipset to detect the actions of game players and use motion-sensing interaction technology to control 3D character models in VR. A similar study ${ }^{(8)}$ showed the construction of a geometric VR platform for environmental navigation, where non-panoramic photos and wearable electronics with Bluetooth wireless transmission functions were used to combine the user's actions with the VR environment in a first-person VR platform. 


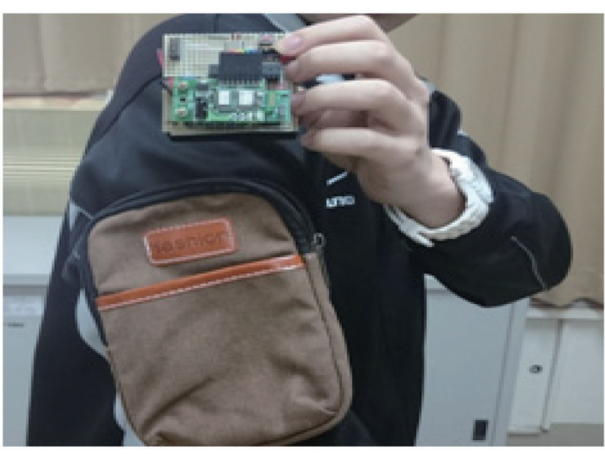

Fig. 13. (Color online) Placing the wearable device into a sports arm sleeve.

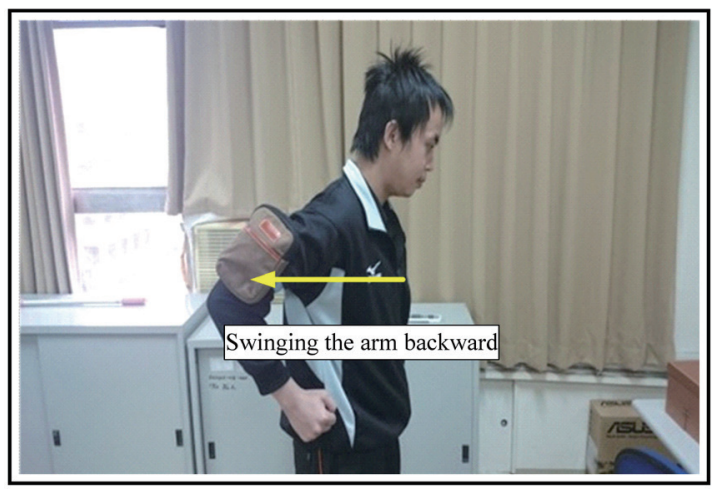

Fig. 15. (Color online) Swinging the arm backward.

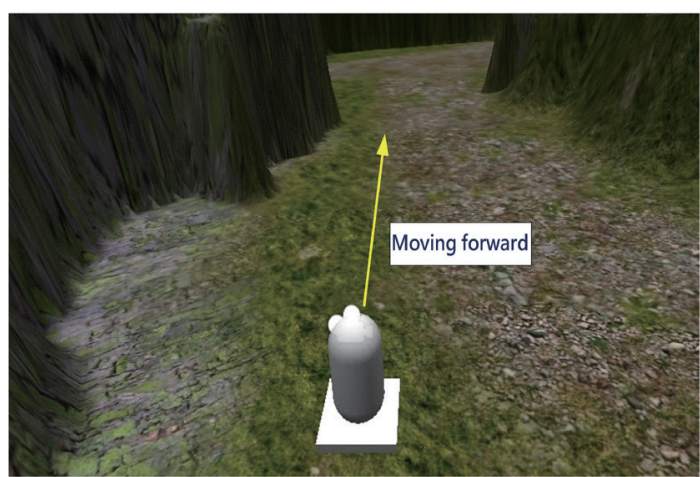

Fig. 17. (Color online) First-person character controller moves forward.

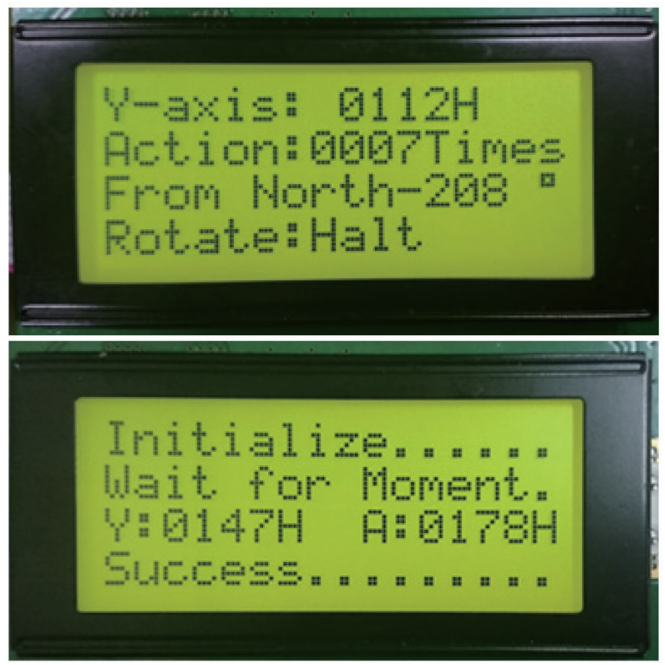

Fig. 14. (Color online) Receiving initialization and test data.

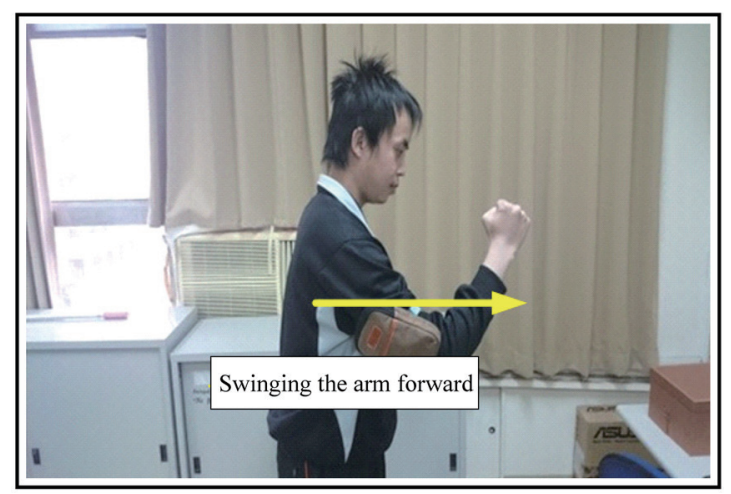

Fig. 16. (Color online) Swinging the arm forward.

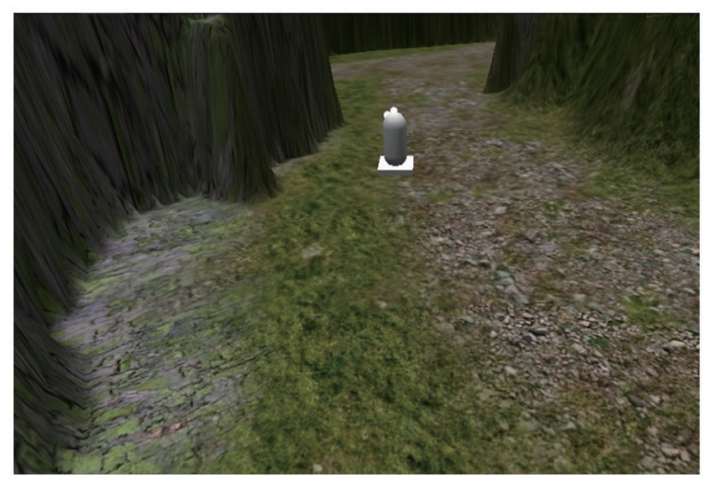

Fig. 18. (Color online) Character controller moves forward to the valley exit. 


\section{Conclusions}

In the study, we used an electronic compass to detect the directions of rotation of players in a VR game and utilized a three-axis accelerometer to detect the movements of the players. The Unity 3D game development engine was used to design the game script, and modeling materials from Maya and 3ds Max were imported into Unity 3D to enrich the model materials of game scenes and enhance the appearance and flexibility of the game. For example, ancient Chinese palaces, pavilions, houses, and animals were added to the game scenes. Unity can now release the game on various device platforms, which can then be used to develop cross-platform games for platforms such as PC, PS3, and Android. Unity provides a very user-friendly interface and supports game script development in C\# or Java to significantly simplify the development process so that users can easily develop games.

\section{Acknowledgments}

The authors would like to thank the Ministry of Science and Technology, ROC, for financially supporting this research under Contract No. MOST 108-3116-F-002-009-CC2.

\section{References}

1 Q. Zhang, G. Chen, C. Y. Chang, and C. C. Chen: J. Internet Technol. 19 (2018) 391. https://doi.org/10.3966/16 0792642018031902008

2 C. T. Chiang, C. M. Chang, and C. C. Chang: IEEE Sens. J. 18 (2018) 4673. https://doi.org/10.1109/ JSEN.2018.2828023

3 J. E. Park and Y. H. Park: J. Commun. Networks 20 (2018) 279. https://doi.org/10.1109/JCN.2018.000040

4 S. Son, H. So, J. Kim, D. Choi, and H. J. Lee: Electron. Lett. 52 (2016) 113. https://doi.org/10.1049/el.2015.2959

5 W. F. Wu, P. S. Tsai, N. T. Hu, J. Y. Chen, and J. J. Jheng: J. Internet Technol. 20 (2019) 471. https://doi.org/10. 3966/160792642019032002015

6 W. F. Wu, P. S. Tsai, N. T. Hu, J. Y. Chen, and Y. S. Huang: Sens. Mater. 30 (2018) 609. https://doi. org/10.18494/SAM.2018.1787

7 W. F. Wu, H. C. Huang, and Y. R. Chien: Sens. Lett. 11 (2013) 2145. https://doi.org/10.1166/sl.2013.2961

8 N. T. Hu, P. S. Tsai, W. F. Wu, J. Y. Chen, and L. Lee: Adv. Mech. Eng. 10 (2018) 1. https://doi. org $/ 10.1177 / 1687814018783636$

9 J. D. Bayliss and D. H. Ballard: IEEE Trans. Rehabil. Eng. 8 (2000) 188. https://doi.org/10.1109/86.847811

10 M. Schoning and K. Hameyer: IEEE Trans. Magn. 44 (2008) 1422. https://doi.org/10.1109/TMAG.2007.916142 